\title{
Review of: "Burning for grassland pollination: recently burned patches promote plant flowering and insect pollinators"
}

$\mathrm{Cu}^{1}$

1 University of the Chinese Academy of Sciences (UCAS)

Potential competing interests: The author(s) declared that no potential competing interests exist.

The study investigates the effect of time-since-burning on pollinator abundance and diversity in an urban grassland. It finds that fire depression may have negative effect on pollinator conservation. The experiment is well designed. Relevant data are collected and analyzed properly. The conclusion is convincing and meaningful. Furthermore, the manuscript is well prepared, with clear tables and figures.

I suggest to accept it for publication. 